\title{
Liposomal curcumin inhibits Lewis lung cancer growth primarily through inhibition of angiogenesis
}

\author{
LIQIANG WANG ${ }^{1 *}$, JING ZHANG ${ }^{2,3 *}$, LULU CAI $^{2}$, JING WEN $^{2}$, HUASHAN SHI $^{2}$, \\ DAN $\mathrm{LI}^{2}, \mathrm{FUCHUN} \mathrm{GUO}{ }^{2}$ and YONGSHENG WANG ${ }^{3}$ \\ ${ }^{1}$ State Key Laboratory of Biotherapy, West China Hospital and College of Life Science; \\ ${ }^{2}$ State Key Laboratory of Biotherapy and ${ }^{3}$ Department of Thoracic Oncology, West China Hospital, \\ West China Medical School, Sichuan University, Chengdu, Sichuan 610000, P.R. China
}

Received October 21, 2011; Accepted March 23, 2012

DOI: $10.3892 / \mathrm{ol} .2012 .686$

\begin{abstract}
Curcumin has been proven to effectively inhibit tumor growth by both targeting tumor cells and angiogenesis; however, poor water solubility limits further clinical application. In the present study, we prepared water-soluble liposomal curcumin to investigate its anti-tumor effects and the underlying mechanism. The MTT assay was used to test the anti-proliferative activities for the MS1 murine endothelial and LL/2 Lewis lung cancer cell lines. Apoptosis and cell cycle arrest induced by liposomal curcumin were analysed by flow cytometry. Anti-angiogenic agents and the resulting anti-tumor effects were investigated in a murine lung cancer model. Zebrafish were used to investigate the anti-angiogenic effect of liposomal curcumin in the development of embryos. In vitro, liposomal curcumin inhibited the proliferation of MS1 cells and induced cell cycle arrest and apoptosis. Notably, LL/2 cells showed less sensitivity to the liposomal curcumin in vitro. In vivo, the systemic administration of liposomal curcumin resulted in significant inhibition of tumor growth. CD31 immunohistochemical analysis and alginate encapsulation assay revealed that angiogenesis was decreased by liposomal curcumin treatment. Angiogenesis was also suppressed in the development of zebrafish. Liposomal curcumin showed potent inhibitory activity against murine endothelial cells but not lung cancer cells. Liposomal curcumin treatment is capable of significantly inhibiting tumor growth in vivo, a process that may depend primarily on its anti-angiogenic effects. Our study also indicates that liposomal curcumin may be developed not only for cancer therapy, but also for the treatment of other angiogenesis-related diseases.
\end{abstract}

Correspondence to: Dr Yongsheng Wang, Department of Thoracic Oncology, West China Hospital, West China Medical School, Sichuan University, Keyuan Road 4, Chengdu, Sichuan 610000, P.R. China

E-mail: wangys@scu.edu.cn

*Contributed equally

Key words: liposomal curcumin, angiogenesis, anti-angiogenic agents, lung cancer

\section{Introduction}

Curcumin has been proven to be a promising anti-cancer drug by induction of apoptosis and apoptosis-independent death, and inhibition of proliferation and angiogenesis (1-4). Phase I and II studies of this compound have shown that curcumin is well tolerated and is effective for cancer patients; however, its benefits may be attenuated due to its low bioavailability through oral administration for non-gastrointestinal cancers $(5,6)$. Therefore, novel strategies are required to overcome these limitations, which are mostly due to the low water solubility and low stability of curcumin against gastrointestinal fluids (7). Investigators have recognized that liposomes have the advantage of improving water insolubility and enhancing delivery efficacy of drugs (8). At present, various methods have been reported for the preparation of liposomes (9). Curcumin acts as an anticancer drug through multiple mechanisms; however, the activity of curcumin may be changed in a liposomal form. Furthermore, the water solubility of liposomal curcumin provides a new strategy for intravenous administration. It is speculated that, systemically, intravenous administration may exhibit marked inhibitory effects due to circumvention of the first-pass effect.

Angiogenesis, the process by which capillaries sprout from pre-existing vasculature, is a hallmark of the majority of solid tumors (10). Targeting tumor vascular endothelial cells has proven to be an effective therapeutic strategy in anti-tumor treatment (11). Therefore, anti-angiogenic agents have gained increasing importance in cancer research. Mounting evidence indicates that curcumin inhibits carcinogenesis in various organs and that the common link between these actions is its anti-angiogenic effect (4). Although the anti-cancer and anti-angiogenic effects of curcumin have been evaluated comprehensively, the anti-angiogenic effect of the liposomal form of these compounds has not been extensively studied, particularly when the liposomal curcumin is administered intravenously.

Previously, we developed water-soluble liposomal curcumin with the ethanol injection method. In the current study, we examined the anti-angiogenic and anti-cancer effects of liposomal curcumin on Lewis lung cancer in vitro and in vivo. Our study indicated that the liposomal curcumin primarily inhibits 
tumor growth due to its anti-angiogenic activity. Our results also indicate that liposomal curcumin may be used in tumor treatment for further clinical application.

\section{Materials and methods}

Cell lines and animals. Murine Lewis lung carcinoma cell line LL/2 and endothelial cell line MS1 were purchased from the American Type Culture Collection (Manassas, VA, USA). The cell lines were cultured in DMEM supplemented with $10 \%$ (vol/vol) fetal bovine serum and were maintained in a humidified chamber at $37^{\circ} \mathrm{C}$ in $5 \% \mathrm{CO}_{2}$ atmosphere. $\mathrm{C} 57 \mathrm{BL} / 6$ and zebrafish (FLK-1:EGFP) were purchased from the West China Experimental Animal Center. The study protocol was reviewed and approved by the institutional animal care and treatment committee of Sichuan University, Chengdu, China.

Cell proliferation assay. The MTT assay was performed to determine the effect of curcumin on MS1 and LL/2 viability. Briefly, cells were plated in a 96-well plate at a density of 3000 cells per well and were exposed to liposomal curcumin at different concentrations for $48 \mathrm{~h}$. Cells grown in media without curcumin were used as a control. Following treatment, the media were carefully removed. Then, $20 \mu \mathrm{l}$ MTT $(5 \mathrm{mg} / \mathrm{ml})$ was added to each well and incubated with the cells for $3 \mathrm{~h}$. Dimethyl sulfoxide $(150 \mu \mathrm{l})$ was added to each well and the plates were read at $570 \mathrm{~nm}$ in an ELISA reader.

Flow cytometry. The percentage of apoptotic cells and the cell cycle distribution of curcumin-treated cells were analyzed by flow cytometry. Briefly, cells $\left(1 \times 10^{5} /\right.$ well $)$ were plated in 6 -well plates. Following incubation overnight, the cells were treated with various concentrations of liposomal curcumin $(0-40 \mu \mathrm{g} / \mathrm{ml})$ for $48 \mathrm{~h}$, trypsinized and washed with PBS, and centrifuged. Supernatants were removed and the cells were resuspended in $1 \mathrm{ml}$ of hypotonic fluorochrome solution containing $50 \mathrm{mg} / \mathrm{ml}$ propidium iodide in $0.1 \%$ sodium citrate plus $0.1 \%$ Triton $\mathrm{X}-100$ and immediately subjected to flow cytometry (ESP Elite, Beckman Coulter Fullerton, CA, USA).

Tumor growth inhibition experiment in vivo. Six-week-old female C57BL/6 mice were acclimatized for one week and fed with animal chow and water ad libitum. The mice were injected subcutaneously in the right leg with $5 \times 10^{5}$ Lewis lung carcinoma cells with a total volume of $50 \mu 1$. Seven days later, when the tumors were palpable, the mice were randomized into two groups ( $\mathrm{n}=6$ per group). The experimental group was treated with liposomal curcumin $(10 \mathrm{mg} / \mathrm{kg})$ by intravenous injection once a day for two weeks. The control mice were administered normal saline (NS). Tumor dimensions were measured every three days with calipers. Tumor volume was calculated according to the formula: volume $=$ width $^{2} \mathrm{x}$ length $\mathrm{x} 0.52$.

Detection of microvessel density. Frozen sections of the tumor tissue from the mice were used to determine vessel density with an anti-CD31 antibody, as described in a previous study in detail (12). The following antibodies and reagents were used: monoclonal rat anti-mouse CD31 antibody (1:400, Santa Cruz Biotechnology, Santa Cruz, CA, USA),

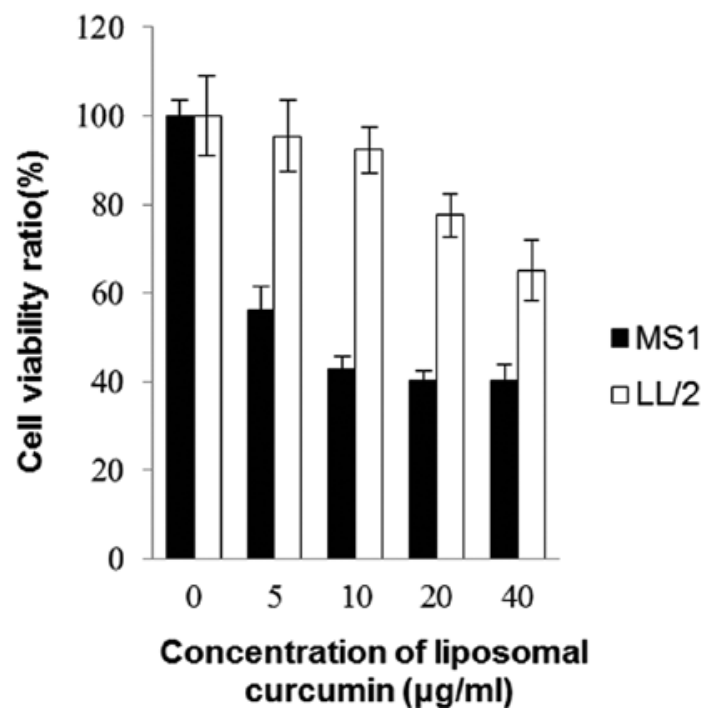

Figure 1. The effects of curcumin on cell viability were examined using the MTT assay. The values for each concentration tested are the average (mean $\pm \mathrm{SD}$ ) from six replicate wells. Curcumin had higher cytotoxic activity against MS1 compared with LL/2 $(\mathrm{P}<0.05)$.

biotinylated polyclonal goat anti-rat antibody (1:200, Vector Laboratories, Peterborough, UK), ABC kit (Boster Biological Engineering Co., Wuhan, China) and DAB visualization system (ZSJQ Biotechnology, Beijing, China). Sections were counterstained with hematoxylin and mounted with glass coverslips. Images were captured using an Olympus fluorescence microscope at an original magnification of x200. Microvessel density (MVD) was assessed within hot spots.

Alginate encapsulation for tumor cell assay. Alginate beadcontaining tumor cell assays were described in detail in a previous study (13). Briefly, cultured LL/2 cells were resuspended with $1.5 \%(\mathrm{~m} / \mathrm{v})$ sodium alginate (Sigma-Aldrich, St. Louis, MO, USA). The tumor cell alginate solution was then dropped into a swirling bath of $0.25 \mathrm{M} \mathrm{CaCl}_{2}$ in order to form droplets containing approximately $1 \times 10^{5}$ tumor cells per bead. After being anesthetized, the C57BL/6 mice were implanted subcutaneously with four beads through an incision on the back and the incisions were sutured with surgical clamps. Treatment with liposomal curcumin $(10 \mathrm{mg} / \mathrm{kg})$ was performed once per day following bead implantation, with normal saline (NS) as a control. At 14 days, the mice were injected intravenously with $100 \mu$ FITC-dextran solution (Sigma Chemical) $(100 \mathrm{mg} / \mathrm{kg})$ and were sacrificed $20 \mathrm{~min}$ later. Images of the alginate implants were captured using a SPOT FIEX camera. Alginate beads were transferred to tubes containing $2 \mathrm{ml}$ of saline. The tubes were mixed in a vortex for $20 \mathrm{sec}$ and centrifuged ( $3 \mathrm{~min} ; 1000 \mathrm{x} \mathrm{g}$ ). Finally, the fluorescence of the supernatant was measured to quantify blood vessel formation.

Zebrafish embryo development assay. FLK-1 promoter EGFP transgenic zebrafish (FLK-1:EGFP) were used. Fertilized eggs were incubated for $8 \mathrm{~h}$, after which liposomal curcumin was added to the water at a concentration of $5 \mu \mathrm{g} / \mathrm{ml}$. The same water without curcumin was used as a control. Following incubation for $72 \mathrm{~h}$, the larvae were placed on glass slides 

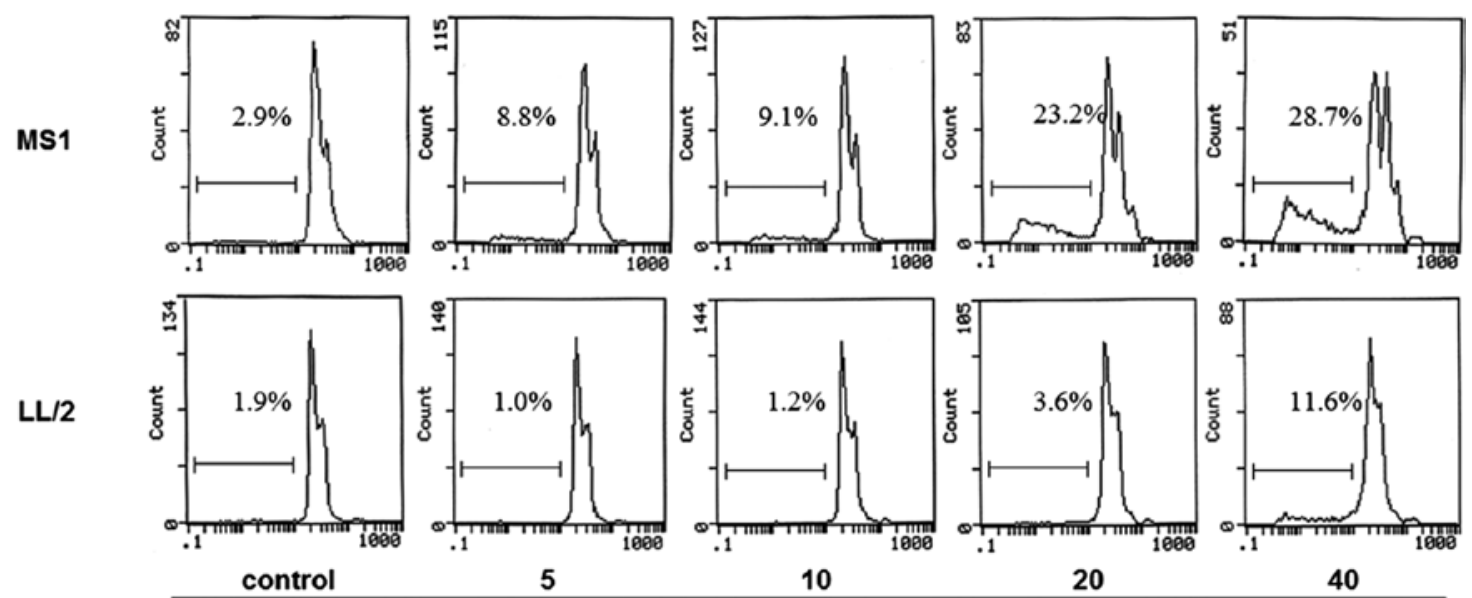

Concentration of liposomal curcumin $(\mu \mathrm{g} / \mathrm{ml})$

Figure 2. Effects of liposomal curcumin on cell apoptosis. Following treatment with liposomal curcumin at various concentrations for $48 \mathrm{~h}$, apoptotic MS1 and LL/2 cells were assessed by flow cytometry.

and examined using a Zeiss microscope. Fluorescence signals were detected and images were captured.

\section{Results}

Higher sensitivity to liposomal curcumin in murine endothelial cell line MS1. An MTT assay was conducted on murine Lewis lung carcinoma cell line LL/2 and endothelial cell line MS1. Fig. 1 shows the effects of liposomal curcumin on cell viability following $48 \mathrm{~h}$ of drug exposure. The cell viability decreased in each cell line with the increasing concentration of curcumin treatment. The sensitivity to curcumin differed markedly between MS1 and LL/ 2 cells. Curcumin had higher cytotoxic activity towards MS1, but lower cytotoxic activity towards LL/2 $(\mathrm{p}<0.05)$. It was clear that mouse endothelial cells were more sensitive to curcumin in comparison to Lewis lung cancer cells. In addition, flow cytometry was performed to investigate whether liposomal curcumin induced MS1 and LL/2 cell apoptosis. The quantitative assessment of sub-G1 cells by flow cytometry was used to estimate the number of apoptotic cells. Liposomal curcumin was found to increase the number of sub-G1 cells compared with the control groups (Fig. 2). Notably, no marked pro-apoptotic effect of liposomal curcumin was observed against LL/2 cells.

Changes of cell cycle phase distribution mediated by liposomal curcumin. To evaluate the cell cycle phase distribution of MS1 and LL/2 cells with curcumin treatment, the DNA content was measured using flow cytometry. FACS analysis of MS1 cells revealed that exposure to liposomal curcumin from 5 to $40 \mu \mathrm{g} /$ $\mathrm{ml}$ for $48 \mathrm{~h}$ caused an increase of the G2/M-phase population from 15.8 to $37.8 \%$, compared to control cells with $16.2 \% \mathrm{G} 2 / \mathrm{M}$ phase cells (Fig. 3A). This increase was accompanied by a significant decrease in the percentage of S-phase cells, whereas the fraction of G1-phase cells was mainly unchanged (Fig. 3C). This result demonstrates that curcumin induces growth inhibitory effects on MS1 at least in part via G2/M phase arrest. Results of the FACS analysis of propidium iodide-stained LL/2 cells showed that liposomal curcumin also reduced $\mathrm{S}$ phase and increased $\mathrm{G} 2 / \mathrm{M}$ percentages in LL/2 compared to the control
A
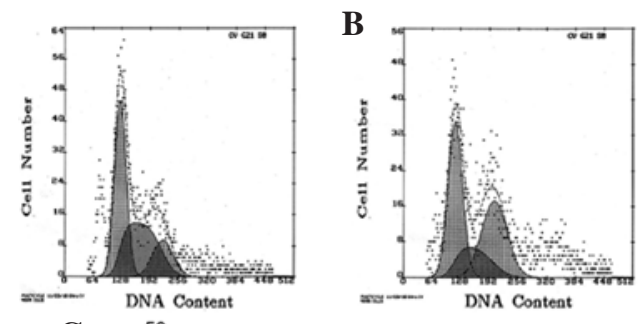

C

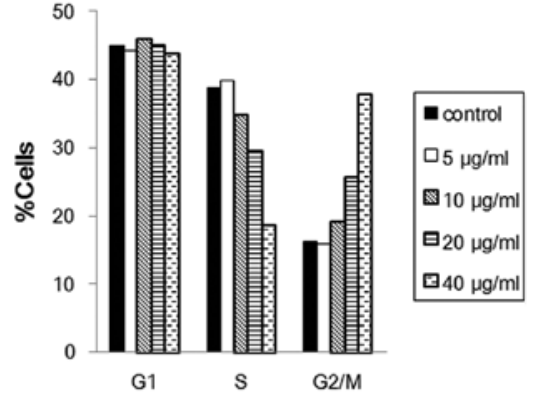

D

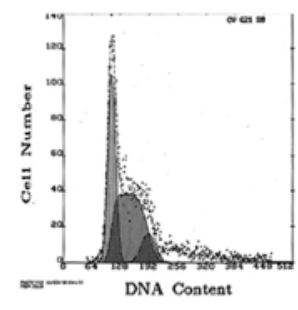

$\mathbf{E}$

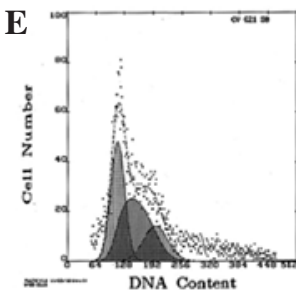

$\mathbf{F}$

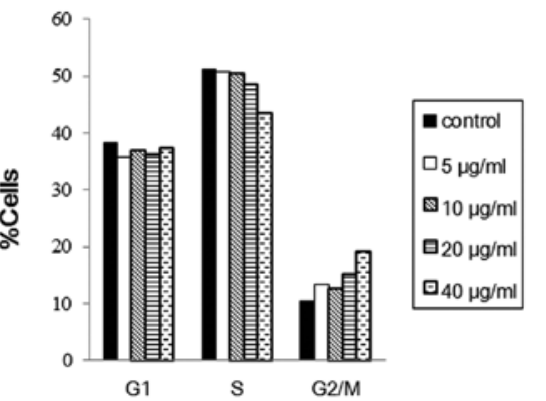

Figure 3. Cell cycle distribution of MS1 and LL/2 cells. Cell cycle distribution of MS1 and LL/2 cells following treatment with various concentrations of curcumin for $48 \mathrm{~h}$. (A) Untreated control MS1 cells; (B) MS1 cells treated with $40 \mu \mathrm{g} / \mathrm{ml}$ curcumin; (C) MS1 cell cycle distribution (percentage of total) of various concentration treatment groups; (D) untreated control LL/2 cells; (E) MS1 cells treated with $40 \mu \mathrm{g} / \mathrm{ml}$ curcumin; (F) LL/2 cell cycle distribution (percentage of total) of different concentration treatment groups. 


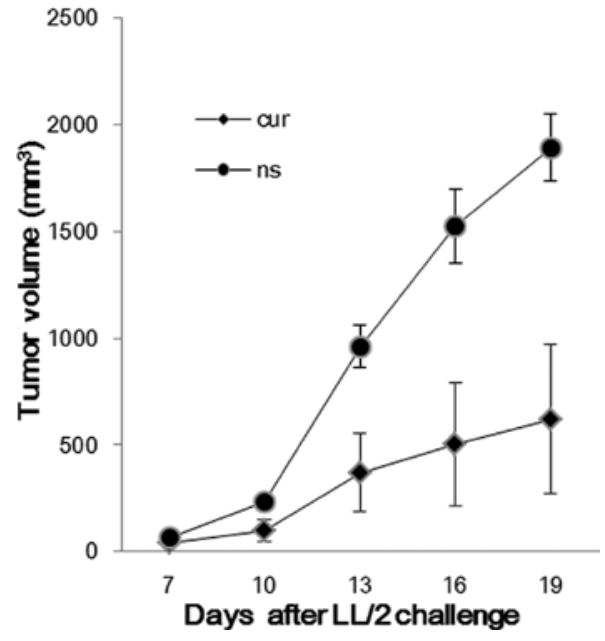

Figure 4. Liposomal curcumin-mediated inhibition of tumor growth in the LL/2 model. Mice were treated intravenously with NS or curcumin $(10 \mathrm{mg} / \mathrm{kg})$ every day. Tumor sizes on each mouse were measured every three days. Treatment with liposomal curcumin resulted in the marked inhibition of tumor growth $(\mathrm{P}<0.05)$.

cells (Fig. 3D). However, the change of cell cycle distribution with the increasing concentration of liposomal curcumin treatment was relatively insignificant in the LL/2 cell line (Fig. 3F).

Tumor growth inhibition in vivo. The established LL/2 tumor model was used to observe the effect of liposomal curcumin on the tumor burden of mice. The treatment regimens were carried out as described in Materials and methods. Compared with the control group, the liposomal curcumin-treated group was found to significantly inhibit tumor growth (Fig. 4). The tumor volume of the control and treated groups was $\left(1892.26 \pm 158.03 \mathrm{~mm}^{3}\right)$ vs. $\left(618.64 \pm 350.26 \mathrm{~mm}^{3}\right)$ on day 19 .

Inhibition of tumor-induced angiogenesis. Tumor sections from each group were stained with anti-CD31 antibody (Fig. 5). Liposomal curcumin treatment resulted in the significant inhibition of angiogenesis in tumors (Fig. 5B) compared with the controls (Fig. 5A). Angiogenesis within tumor tissue was estimated by counting the number of microvessels on the section stained with an antibody reactive to CD31. Tumors from the liposomal curcumin group exhibited lower vessel density than those of the NS group (Fig. 5C). In addition, the inhibition of angiogenesis in vivo was observed through alginate encapsulation assay. Alginate implant angiogenesis was quantitated by measuring the uptake of FITC-dextran into beads. Vascularization of the alginate beads was reduced, and FITC-dextran uptake was decreased in liposomal curcumin-treated mice compared to the controls (Fig. 6).

Liposomal curcumin-mediated anti-angiogenesis in development of the zebrafish embryo. Our data demonstrate that liposomal curcumin effectively inhibited endothelial cell growth in vitro and in vivo. The endothelial cell line MS1 is a pancreatic islet endothelial cell line derived from C57BL/6
$\mathbf{A}$

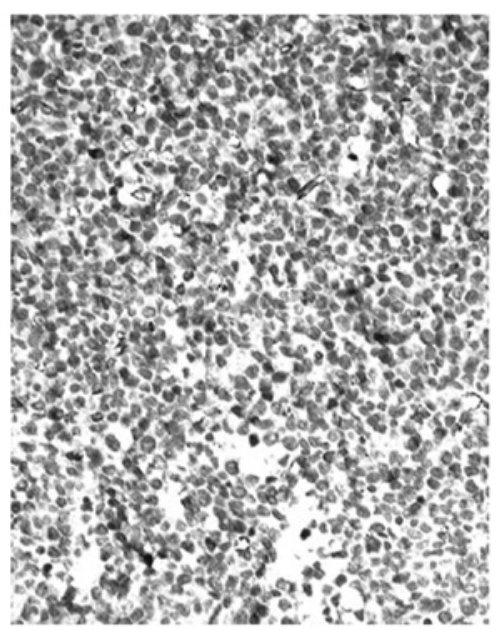

B

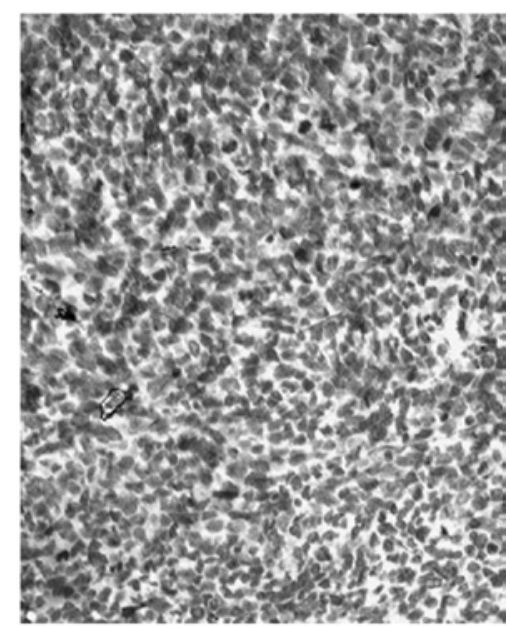

C

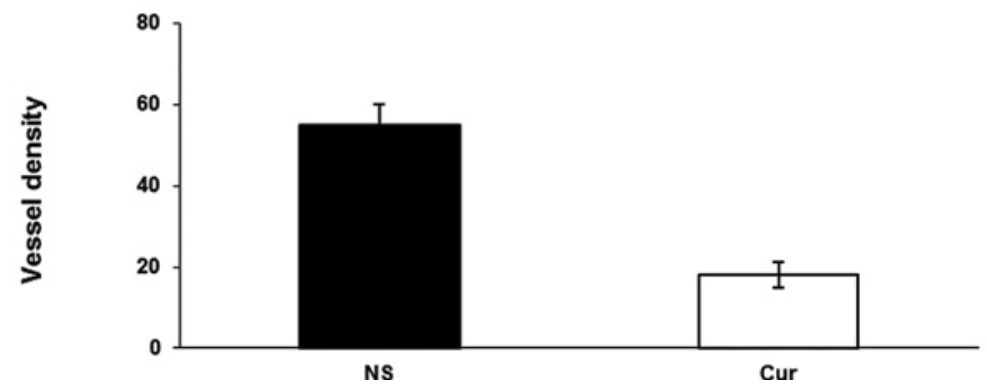

Figure 5. Inhibition of angiogenesis within the tumor estimated by CD31 immunohistochemical analysis. (A) Representative sections from the normal saline (NS) group. (B) Representative sections from the liposomal curcumin (Cur) group. (C) Vessel density was determined by counting the number of the microvessels per high-power field within a hot-spot area. Tumors of the liposomal curcumin group showed a smaller microvessel count than the NS group $(\mathrm{P}<0.05)$. 

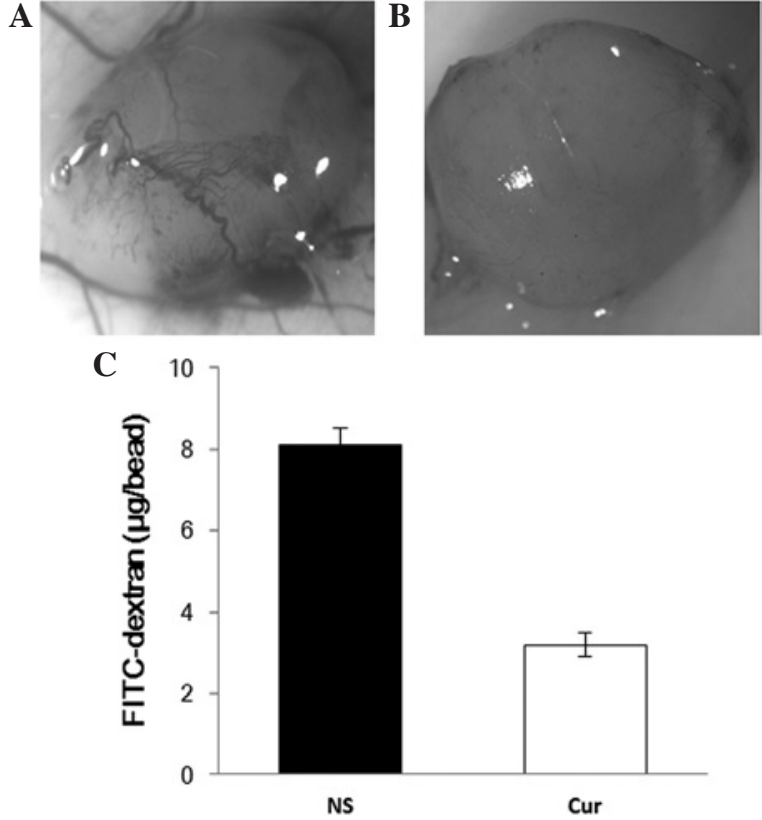

NS

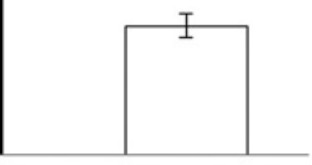

Cur

Figure 6. Inhibition of angiogenesis in tumors was estimated by encapsulation assay. Alginate beads containing $1 \times 10^{5} \mathrm{LL} / 2$ cells were implanted subcutaneously into C57BL/6 mice, which were treated with liposomal curcumin (Cur) once a day.Fourteen days later, beads were surgically removed, and FITC-dextran was quantified as described in Materials and methods. (A) Representative alginate beads from the normal saline (NS) group. (B) Representative alginate beads from liposomal curcumin group. (C) FITC-dextran uptake of beads from each group. The curcumin group showed a significant decrease in vascularization compared with the control group $(\mathrm{P}<0.05)$.

mice and may exhibit physiological and pathological roles in the current C57BL/6 mouse model. Therefore, our data also revealed that the inhibition of angiogenesis mediated by liposomal curcumin is non-specific to tumor angiogenesis. To verify this, the anti-angiogenic effects of liposomal curcumin on the development of the zebrafish embryo were investigated. Larvae hatched from fertilized eggs treated with liposomal curcumin exhibited developmental defects. In the control fish, vascularization was normal. The angiogenetic defects caused by liposomal curcumin were evident compared to the control group (Fig. 7), indicating that inhibition of angiogenesis mediated by liposomal curcumin is non-specific and thus may show toxicity in physiological processes, such as wound healing.

\section{Discussion}

Curcumin inhibits tumor growth by targeting tumor cells and endothelial cells. The current study evaluated the anti-tumor and anti-angiogenesis activity of liposomal curcumin in a Lewis lung cancer model. Since a critical step in angiogenesis involves the proliferation of endothelial cells, we also determined the effect of liposomal curcumin on the viability of murine endothelial cells both in vitro and in vivo. Our data showed that the inhibitory effects of liposomal curcumin on angiogenesis were more effective. Inhibition of the $\mathrm{NF}-\kappa \mathrm{B}$ pathway mediated by curcumin is known to play a key role in its pharmacological activity $(14,15)$. Accumulating evidence has shown that both physiological and pathological angiogenesis rely on the activity of the NF- $\mathrm{BB}$ pathway (16). However,

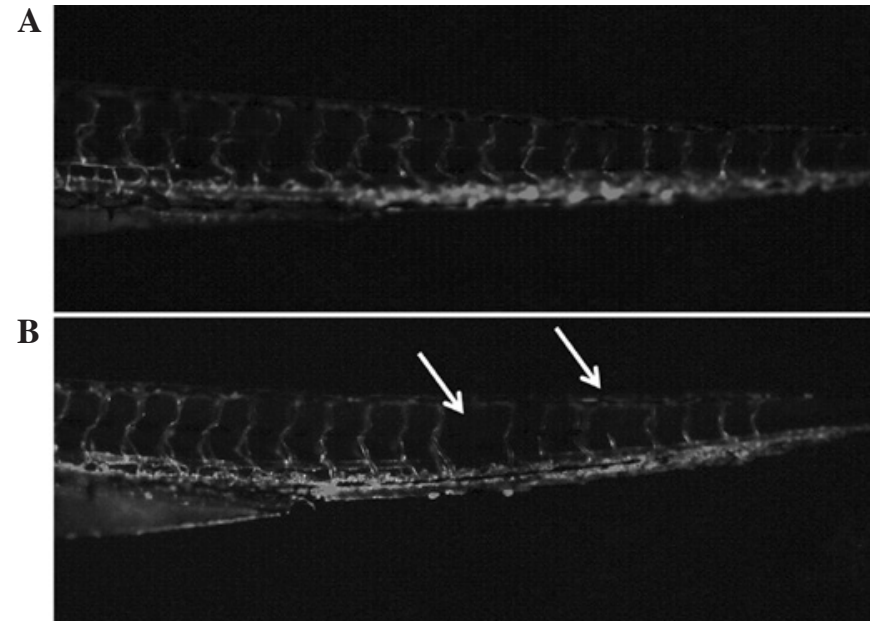

Figure 7. Effects of liposomal curcumin on the development of zebrafish Transgenic FLK-1:EGFP zebrafish larvae were visualized under a Zeiss microscope. (B) Larvae hatching out of the liposomal curcumin-treated fertilized eggs, as indicated by the white arrows, showed a reduction or disappearance of certain vessels compared to the (A) controls.

$\mathrm{NF}-\kappa \mathrm{B}$ signaling has not been a preferred drug target in current clinical cancer therapy since the activity of $\mathrm{NF}-\kappa \mathrm{B}$ signaling is not necessary for tumor growth.

The significant finding of the current study is that murine endothelial cells (MS1) were more sensitive than murine lung tumor cells (LL/2). The cytotoxic activity of curcumin against these two cell lines was evaluated by MTT assay. Following treatment with curcumin ( 5 to $40 \mu \mathrm{g} / \mathrm{ml}$ ) for $48 \mathrm{~h}$, the viability of endothelial cells was markedly inhibited and lung cancer cells by comparison were only slightly inhibited. Moreover, the difference is clearer in low concentrations of curcumin $(<20 \mu \mathrm{g} / \mathrm{ml})$. We further investigated the possible mechanisms of this result. Curcumin inhibits cell proliferation through diverse mechanisms. However, as yet, the exact mechanism is not clear since various mechanisms act on different cells. Two possible mechanisms may have been involved in generating the results of this study. The first is induction of apoptosis: Our study showed that liposomal curcumin induces more cells to apoptosis in MS1 murine endothelial cells than in LL/2 murine lung tumor cells. It is also noteworthy that it had almost no effect on LL/2 at a concentration of $20 \mu \mathrm{g} / \mathrm{ml}$. The possible mechanisms underlying the induction of apoptosis by liposomal curcumin may differ in the two cell lines; a more mechanistic study is therefore required in this area. The second possible mechanism is cell cycle arrest: We report in this study that liposomal curcumin treatment leads to $\mathrm{G} 2 / \mathrm{M}$ arrest in MS1 and LL/2 cell lines. However, the effect on MS1 is more obvious than that on $\mathrm{LL} / 2$. In recent studies, other authors have reported that certain tumor cells are resistant to curcumin $(17,18)$, which may correspond with the different genetics and biologies of individual tumors. The lack of effect of curcumin on LL/2 shown in our current study is consistent with their reports. Therefore, the inhibition of tumor angiogenesis is thought to play a more significant role in its anti-tumor effects. In addition, it is known that endothelial cells are genetically stable, and therefore less likely to rapidly develop drug resistance. Taken together, these results indicate that liposomal curcumin targeting angiogenesis that supports 
tumor growth rather than the tumors themselves is a promising therapeutic approach for certain cancer types.

Various angiogenetic inhibitors have been developed to target vascular endothelial cells and block tumor growth. The majority of these inhibitors have limited potential since they are extremely toxic or highly expensive, and are therefore beyond the reach of most patients. Curcumin, a plant-derived compound, has been used safely as a food additive for centuries without reports of significant toxicity. In addition, curcumin is affordable and has been found to suppress angiogenesis through multiple mechanisms (18). Most of the studies used free curcumin, which is poorly water-soluble and has low bioavailability, and is therefore limited in its clinical efficacy. We prepared liposomal curcumin solution via the ethanol injection method. This solution is well dispersed and shows an average size of $125.7 \mathrm{~nm}$, determined using a nano-particle size analyzer (data not shown). Liposome encapsulation of curcumin renders this agent amenable to intravenous administration.

A particularly encouraging aspect of this study is the observation that liposomal curcumin significantly suppressed LL/2 tumor growth in vivo, although it was almost ineffective on LL/2 cells in vitro. This result indicates that liposomal curcumin can be used for cancer therapy in curcumin-sensitive and -resistant tumor cells. The approach of anti-angiogenesis for the curcumin treatment of cancer appears promising. Our results also indicate that curcumin has the ability to block angiogenesis in vivo. This result may be associated with the inhibition of proliferation and the induction of apoptosis and cell cycle arrest in endothelial cells in vitro. Liposomal curcumin has been reported to exhibit anti-cancer activity on colorectal cancer (19), prostate cancer (20), head and neck squamous cell carcinoma (21), and pancreatic (22) and cervical cancer (23). In this study, we revealed that liposomal curcumin inhibits tumor growth in the Lewis lung cancer mouse model primarily by targeting tumor angiogenesis.

The effect of liposomal curcumin on physiological angiogenesis was also investigated in this study. We used zebrafish as vertebrate model organisms to investigate the anti-angiogenic effect of curcumin in the development of the embryo. Advantages of using zebrafish as model organisms include their fecundity, optical clarity, and genetic similarity to mammals. Research using rats as animal models revealed that orally administered curcumin had no toxic effects on fertility or pregnancy (24). Our findings have shown that, unlike in rats, liposomal curcumin had embryotoxic and anti-angiogenic effects on the development of zebrafish embryos. Therefore, our study indicates that liposomal curcumin-mediated anti-angiogenic effects are not tumor-specific but broad-spectrum and may be used to treat angiogenesis-related diseases. However, further investigation into the toxicity of liposomal curcumin is required.

\section{Acknowledgements}

This study was financially supported by the Chinese National Natural Science Foundation.

\section{References}

1. Pongrakhananon V,Nimmannit U,Luanpitpong S, Rojanasakul Y and Chanvorachote P: Curcumin sensitizes non-small cell lung cancer cell anoikis through reactive oxygen species-mediated Bcl-2 downregulation. Apoptosis 15: 574-585, 2010.
2. Bharti AC, Donato N, Singh S and Aggarwal BB: Curcumin (diferuloylmethane) down-regulates the constitutive activation of nuclear factor-kappa B and IkappaBalpha kinase in human multiple myeloma cells, leading to suppression of proliferation and induction of apoptosis. Blood 101: 1053-1062, 2003.

3. O'Sullivan-Coyne G, O'Sullivan GC, O'Donovan TR, Piwocka K and McKenna SL: Curcumin induces apoptosis-independent death in oesophageal cancer cells. Br J Cancer 101: 1585-1595, 2009.

4. Bhandarkar SS and Arbiser JL: Curcumin as an inhibitor of angiogenesis. Adv Exp Med Biol 595: 185-195, 2007.

5. Cheng AL, Hsu CH, Lin JK, et al: Phase I clinical trial of curcumin, a chemopreventive agent, in patients with high-risk or pre-malignant lesions. Anticancer Res 21: 2895-2900, 2001.

6. Dhillon N, Aggarwal BB, Newman RA, et al: Phase II trial of curcumin in patients with advanced pancreatic cancer. Clin Cancer Res 14: 4491-4499, 2008.

7. Takahashi M, Uechi S, Takara K, Asikin Y and Wada K: Evaluation of an oral carrier system in rats: bioavailability and antioxidant properties of liposome-encapsulated curcumin. J Agric Food Chem 57: 9141-9146, 2009.

8. Allen TM and Cullis PR: Drug delivery systems: entering the mainstream. Science 303: 1818-1822, 2004.

9. Bejjani RA, Jeanny JC, Bochot A and Behar-Cohen F: The use of liposomes as intravitreal drug delivery system. J Fr Ophtalmol 26: 981-985, 2003 (In French).

10. Carmeliet P and Jain RK: Angiogenesis in cancer and other diseases. Nature 407: 249-257, 2000.

11. Gasparini G, Longo R, Toi M and Ferrara N: Angiogenic inhibitors: a new therapeutic strategy in oncology. Nat Clin Pract Oncol 2: 562-577, 2005.

12. Bai RZ, Wu Y, Liu Q, et al: Suppression of lung cancer in murine model: treated by combination of recombinant human endostsatin adenovirus with low-dose cisplatin. J Exp Clin Cancer Res 28: 31, 2009.

13. He QM: Inhibition of tumor growth with a vaccine based on xenogeneic homologous fibroblast growth factor receptor-1 in mice. J Biol Chem 278: 21831-21836, 2003.

14. Zheng M, Ekmekcioglu S, Walch ET, Tang CH and Grimm EA: Inhibition of nuclear factor-kappaB and nitric oxide by curcumin induces $\mathrm{G} 2 / \mathrm{M}$ cell cycle arrest and apoptosis in human melanoma cells. Melanoma Res 14: 165-171, 2004.

15. Liao S, Xia J, Chen Z, et al: Inhibitory effect of curcumin on oral carcinoma CAL-27 cells via suppression of Notch-1 and NF- $\kappa B$ signaling pathways. J Cell Biochem 112: 1055-1065, 2011.

16. Tabruyn SP and Griffioen AW: A new role for NF- $\mathrm{kB}$ in angiogenesis inhibition. Cell Death Differ 14: 1393-1397, 2007.

17. Wang WZ, Cheng J, Luo J and Zhuang SM: Abrogation of G2/M arrest sensitizes curcumin-resistant hepatoma cells to apoptosis. FEBS Lett 582: 2689-2695, 2008.

18. Yadav VR and Aggarwal BB: Curcumin: a component of the golden spice, targets multiple angiogenic pathways. Cancer Biol Ther 11: 236-241, 2011.

19. Wang W, Bernard K, Li G and Kirk KL: Curcumin opens cystic fibrosis transmembrane conductance regulator channels by a novel mechanism that requires neither ATP binding nor dimerization of the nucleotide-binding domains. J Biol Chem 282: 4533-4544, 2007.

20. Thangapazham RL, Puri A, Tele S, Blumenthal R and Maheshwari RK: Evaluation of a nanotechnology-based carrier for delivery of curcumin in prostate cancer cells. Int J Oncol 32: $1119-1123,2008$

21. Wang D, Veena MS, Stevenson K, et al: Liposome-encapsulated curcumin suppresses growth of head and neck squamous cell carcinoma in vitro and in xenografts through the inhibition of nuclear factor kappaB by an AKT-independent pathway. Clin Cancer Res 14: 6228-6236, 2008.

22. Mach CM, Mathew L, Mosley SA, Kurzrock R and Smith JA: Determination of minimum effective dose and optimal dosing schedule for liposomal curcumin in a xenograft human pancreatic cancer model. Anticancer Res 29: 1895-1899, 2009.

23. Sreekanth CN, Bava SV, Sreekumar E and Anto RJ: Molecular evidences for the chemosensitizing efficacy of liposomal curcumin in paclitaxel chemotherapy in mouse models of cervical cancer. Oncogene 30: 3139-3152, 2011.

24. Ganiger S, Malleshappa HN, Krishnappa H, Rajashekhar G, Ramakrishna Rao V and Sullivan F: A two generation reproductive toxicity study with curcumin, turmeric yellow, in Wistar rats. Food Chem Toxicol 45: 64-69, 2007. 